\title{
Effect of Eccentric Distance on Successive Dual-droplet Impacting a Super-hydrophobic Tube
}

\author{
Kaimin Wang, Han Chen, Jiawei Liu, Hongyu Ge, Hongsheng Liu and Xiaohua Liu* \\ Key Laboratory of Ocean Energy Utilization and Energy Conservation of Ministry of Education, \\ School of Energy and Power Engineering, Dalian University of Technology, Dalian 116024, China.
}

\begin{abstract}
Droplet impact is a common but significant phenomenon in industry. The CLSVOF (coupled level set and volume-of-fluid) method is used to numerically study the successive dual-droplet impacting a super-hydrophobic tube. For the impact velocity of $1.0 \mathrm{~m} / \mathrm{s}$, the effect of the eccentric distance on dynamic characteristics is analysed, the corresponding eccentric distances are $0.5,1.0$ and 2.0, respectively. In addition, the break-up during rebound is analysed with velocity field and pressure nephogram. Results show that, the eccentric distance hinders the spread during the initial period of spreading. With the increase in eccentric distance, more liquid gathers at the eccentric side and the liquid film might rebound easily without break-up under the same impact velocity. The break-up during rebound mainly depends on the local airflow and pressure difference. The high-pressure zone near the solid-liquid interface moves towards the eccentric side with the increase in eccentric distance.
\end{abstract}

\section{Introduction}

Droplet impact is a common but significant phenomenon in daily life and industry. The studies on it have been conducted by many scholars [1-5], the majority of which is about single-droplet impact. While in most industrial applications, e.g., spray cooling, multi-droplet impact occurs. The study of multi-droplet impact is important due to the interactions among droplets.

Fujimoto et al. [6] conducted pioneering experiments on successive dual-droplet impacting a flat solid surface. The vertical droplet spacing significantly influences the dynamic behaviours. The spreading factor of dual-droplet is larger than that of single droplet under the same condition. Later, they numerically studied the dual-droplet impact, in which the numerical data using dynamic contact angle model is consistent with the experimental data well. They found the maximum spread factor increases with the increment in impact velocity [7]. The size of liquid crown is mainly influenced by the vertical droplet spacing [8]. Liu et al. [9] numerically studied the successive dual-droplet impact of heptane droplets. They found the height of liquid crown increases with the increase in Reynold number, Weber number and vertical droplet spacing. An

\footnotetext{
* Corresponding author: 1xh723@dlut.edu.cn
} 
incompressible SPH method is adopted to study the multi-droplet impacting a liquid film numerically [10]. A swelling liquid part forms around the bottom of the second droplet if the vertical droplet spacing is large enough. The successive impact of dual-droplet on super-hydrophobic tubes was studied by Chen et al. [11]. The increase in vertical droplet spacing changes the model of droplet impact and promotes break-up of liquid film during the rebound, so does the increase in impact velocity [12]. Liang et al. [13] simulated the multi-droplet impacting a hot liquid film, using a three-dimensional model. It was found, with the increment in initial film thickness and Weber number, the diameter of primary crown decreases while the height of it increases. When droplet impacts a hot liquid film, the average heat flux increases with the increment in impact velocity. The influences of the liquid film thickness, droplet diameter and vertical droplet spacing on average heat flux are little [14]. Recently, Liang et al. [15] discovered the cooling of the surface is determined by the contacting of the trailing droplet with surface, rather than the mixing of heated liquid film and impacting droplets. The decrease in vertical droplet spacing promotes the temperature uniformity on the liquid crown walls [16].

In general, compared with the studies on single droplet impact, the studies on successive multi-droplet impact are seldom, of which are mainly on a flat surface or liquid film. While for some practical applications, the study on multi-droplet impacting curved solid surfaces is important. In this paper, the CLSVOF method is adopted to simulate the process of successive dual-droplet impacting a super-hydrophobic tube, investigating the influence of eccentric distance.

\section{Numerical simulation}

Figure 1 shows the schematic of the dual-droplet impact on a tube. The moment of leading droplet impacting the tube is defined as $0 \mathrm{~ms}$ and the time after droplet impact is $t(\mathrm{~mm})$. The droplet impacting the tube earlier is the leading droplet, the next one is the trailing droplet. The diameter of the leading droplet is $d_{\mathrm{p} 1}(\mathrm{~mm})$ and that of the trailing droplet is $d_{\mathrm{p} 2}$ $(\mathrm{mm})$, the corresponding impact velocities are $v_{\mathrm{p} 1}$ and $v_{\mathrm{p} 2}(\mathrm{~m} / \mathrm{s})$, respectively. The spreading diameter of liquid film is $d(\mathrm{~mm})$, vertical droplet spacing is $S(\mathrm{~mm})$, centre height or rebound height of the liquid film is $h(\mathrm{~mm})$, eccentric distance of droplets is $e$ and diameter of tube is $D(\mathrm{~mm})$, as shown in Figure 1.

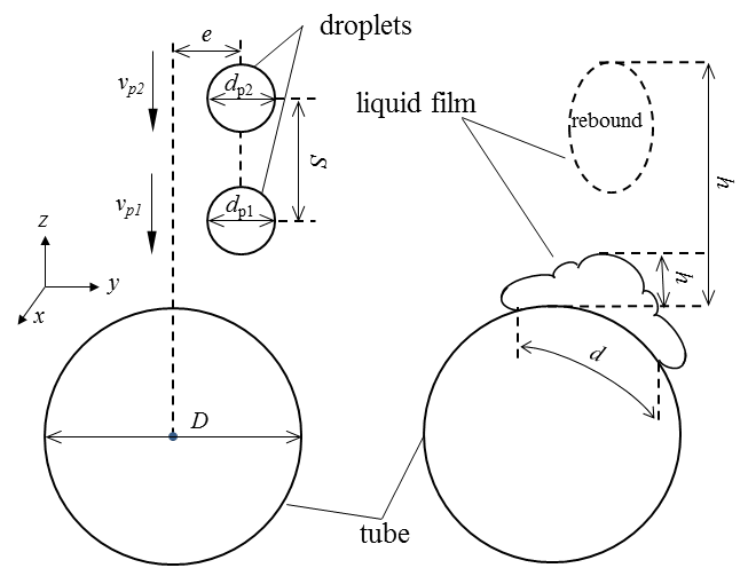

Fig. 1. Schematic of the dual-droplet impact.

The spreading factor is $D^{*}$, dimensionless time is $t^{*}$ and curvature ratio is $\varepsilon$, calculated by equations (1) - (3) [17],[18]. 


$$
\begin{aligned}
& D^{*}=d / d_{\mathrm{p} 1} \\
& t^{*}=t \cdot v_{\mathrm{p} 1} / d_{\mathrm{p} 1} \\
& \varepsilon=d_{\mathrm{p} 1} / D
\end{aligned}
$$

The water and air used in this paper are considered as incompressible Newtonian fluids. In addition, the corresponding density, viscosity and surface tension are considered to be constant. The initial shape of droplets are set to be spherical, the corresponding surfaces are considered as the free surface and the liquid film flow after impacting is considered to be laminar. Furthermore, the temperature of tube surface, droplet and ambient air are considered to be equal, and no heat transfer occurs during droplet impact.

The gas-liquid interface is captured by CLSVOF method. The continuity and Navier-Stokes equations for the incompressible flow are shown in equations (4) - (5) respectively.

$$
\begin{gathered}
\nabla \cdot \mathbf{u}=0 \\
\frac{\rho \partial \mathbf{u}}{\partial t}+\rho \mathbf{u} \cdot \nabla \mathbf{u}=-\nabla p+\nabla \cdot \mu\left[\nabla \mathbf{u}+(\nabla \mathbf{u})^{T}\right]-\sigma k \delta(\phi) \nabla \phi+\rho g
\end{gathered}
$$

where $k$ is interface curvature.

$$
k=\nabla \cdot \frac{\nabla \phi}{|\nabla \phi|}
$$

where $\phi$ is distance function. The continuum surface force (CSF) method is adopted to calculate surface tension [19]:

$$
\begin{gathered}
F=\sigma k \delta(\phi) \nabla \phi \\
\delta(\phi)=\left\{\begin{array}{cc}
\frac{1+\cos (\pi \phi / a)}{2 a} & |\phi|<a \\
0 & |\phi| \geq a
\end{array}\right.
\end{gathered}
$$

where $a$ equals $1.5 \omega$ and $\omega$ is the spacing of grid.

The fluid viscosity and density are determined in equations (9) - (10). Equation (11) shows the definition of Heaviside function.

$$
\begin{gathered}
\rho(\phi)=\rho_{g}+\left(\rho_{l}-\rho_{g}\right) H(\phi) \\
\mu(\phi)=\mu_{g}+\left(\mu_{l}-\mu_{g}\right) H(\phi) \\
H(\phi)= \begin{cases}0 & \phi<-a \\
\frac{1}{2}\left[1+\frac{\phi}{a}-\frac{1}{\pi} \sin \left(\frac{\pi \phi}{a}\right)\right] & |\phi| \leq a \\
1 & \phi>a\end{cases}
\end{gathered}
$$

The verification of tube length and grid independence were conducted and the numerical results were compared with the experimental ones (see Ref. [20] for details), the numerical results agrees well with the experimental ones. 


\section{Results and discussions}

\subsection{Effect of the eccentric distance}

In this paper, the eccentric distances are $0.5,1$ and $2 \mathrm{~mm}$, respectively, $d_{\mathrm{p} 1}$ and $d_{\mathrm{p} 2}$ are $2 \mathrm{~mm}$, $v_{\mathrm{p} 1}$ and $v_{\mathrm{p} 2}$ are $1 \mathrm{~m} / \mathrm{s}, S$ is $3 \mathrm{~mm}, D$ is $6 \mathrm{~mm}(\varepsilon=0.33)$ and $C A$ is $160^{\circ}$.

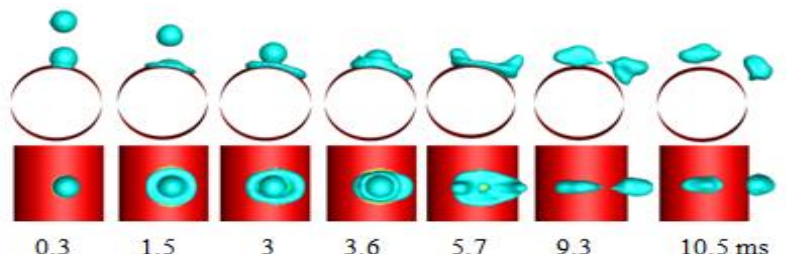

(a)

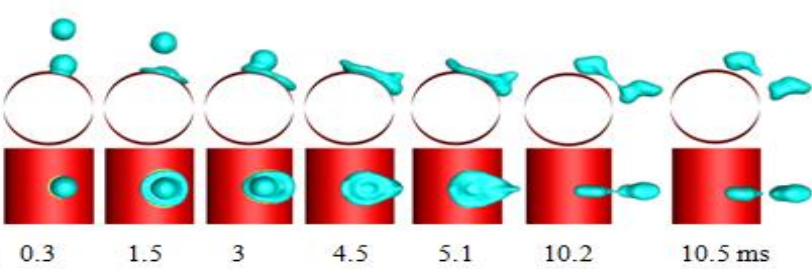

(b)

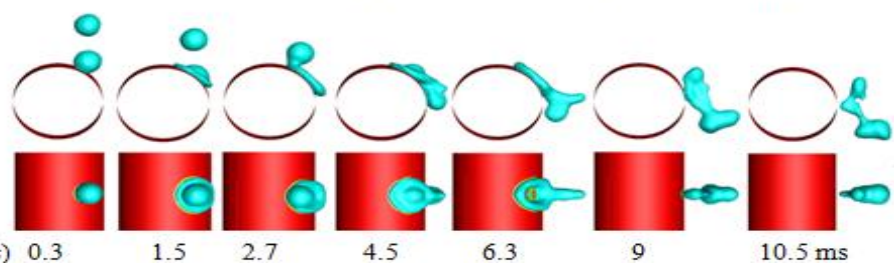

Fig. 2. The dynamic characteristics of dual-droplet impacting a super-hydrophobic tube under different eccentric distances $\left(v_{\mathrm{p} 1}=v_{\mathrm{p} 2}=1 \mathrm{~m} / \mathrm{s}\right)$ : (a) $e=0.5 \mathrm{~mm}$; (b) $e=1 \mathrm{~mm}$; (c) $e=2 \mathrm{~mm}$.

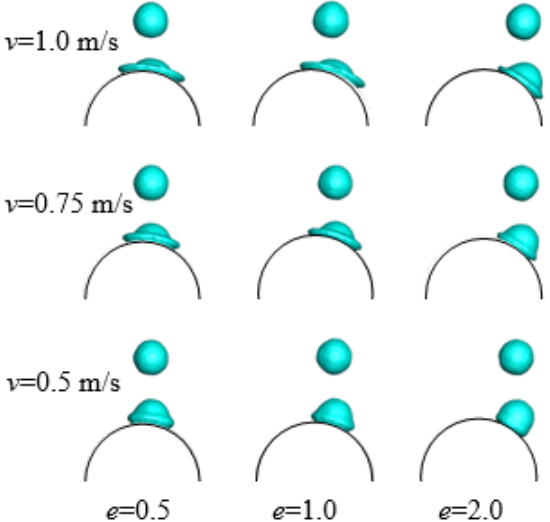

Fig. 3. The dynamic characteristics during the initial period $(t=1.5 \mathrm{~ms})$.

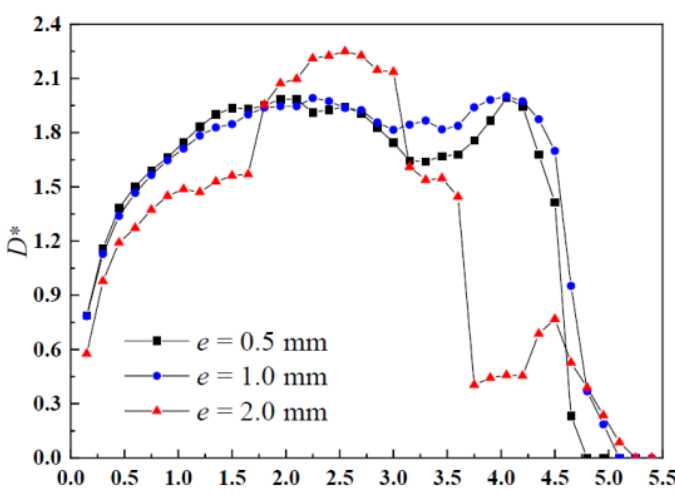

Fig. 4. The changes of spreading factor versus dimensionless time.

Figure 2 shows the dynamic characteristics of dual-droplet impact $\left(v_{\mathrm{p} 1}=v_{\mathrm{p} 2}=1 \mathrm{~m} / \mathrm{s}\right)$. With the increase in eccentric distance, the liquid gathers more at the right rim of the liquid film (eccentric side) with the trailing droplet spread. After impact, the flow inside liquid film experiences downward-radial-upward redirections [15]. For an eccentric impact, it is more difficult for the liquid at eccentric side to flow upward and then retract. Instead, it 
gathers at the liquid film rim of eccentric side due to the gravity. The larger the eccentric distance is, the more the liquid gathers. Thereafter, the liquid film retraction in the axial direction is faster than that in the circumferential direction. This is because the liquid gathering at the liquid film rim hinders the liquid film retraction in the circumferential direction. After retraction, the liquid film rebounds. For the eccentric distance of 0.5 or 1 $\mathrm{mm}$, the liquid film breaks up during rebound while not for the eccentric distance of $2 \mathrm{~mm}$. For the eccentric distances studied, the liquid film break-up during rebound might be hindered with the increase in eccentric distance. The break-up during rebound will be discussed in part 3.2 with velocity field and pressure nephogram. It can also be found, the eccentric distance might hinder the droplet spread during the initial period of spreading (1.5 $\mathrm{ms})$. Figure 3 shows the dynamic characteristics at $1.5 \mathrm{~ms}$ under different impact velocities and eccentric distances. With the increase in eccentric distance, the spreading diameters all become smaller during the initial period of spreading. When the droplet impacts a surface, a transient impact force perpendicular to the impact point develops at the solid-liquid interface [21], causing a high-pressure zone around the impact area forms, which drives liquid film to spread along the surface continually [22]. Through force analysis, the impact force normal to the tube becomes smaller with the increase in eccentric distance under the same impact velocity. Thus the spread of liquid film during the initial period becomes smaller, as shown in Figure $4\left(t^{*}<1.65\right)$.

Figure 4 shows the changes of spreading factor under different eccentric distances. During the initial period of spreading, the spreading factor decreases with the eccentric distance at the same $t^{*}$ (less than 1.65). For the eccentric distance of 0.5 or $1 \mathrm{~mm}$, the spreading factor increases first with the leading liquid film spread and decreases due to retraction. Then it increases again because of a further spread, which is driven by the trailing droplet. The spreading factor sharply decreases to zero eventually because of rebound. For the eccentric distance of $2 \mathrm{~mm}$, the spreading factor increases first with the leading liquid film spread. When $t^{*}$ is less than 1.65 , it's a bit smaller than that for the eccentric distance of 0.5 or $1 \mathrm{~mm}$. For the eccentric distance of $2 \mathrm{~mm}$, more liquid flows to the eccentric side under gravity and the spread of the left part of liquid film is hindered. Thus, the spreading factor is a little smaller. Thereafter the spreading factor fast increases. As mentioned above, for the eccentric distance of $2 \mathrm{~mm}$, more liquid flows to the eccentric side under gravity and gathers. The gravity of the gathering liquid can't be neglected, especially after the trailing droplet impact, causing a further spread of eccentric side when surface tension is overcome by gravity. Then, the spreading factor decreases sharply due to the right-slip and up-warp of the liquid film. Part of the liquid film that up-warps contacts the tube again and the spreading factor increases. Eventually, the spreading factor decreases to zero due to the rebound.

\subsection{Break-up during rebound}

As shown in Figure 2, break-up during rebound occurs under the eccentric distance of 0.5 and 1.0. The mechanism of break-up during rebound is analyzed by velocity field and pressure nephogram, the corresponding cross-section is made at middle of the tube, perpendicular to the axis of the tube.

Figure 5 shows the velocity field and pressure nephogram $(e=0.5 \mathrm{~mm})$. A high-pressure zone occurs at the bottom of liquid film $(8.1 \mathrm{~ms})$, which promotes the liquid film flow outside and upward around the high-pressure zone [22]. During spread, the liquid film forms two liquid necks $(8.7 \mathrm{~ms})$, pointed by arrows. The upper liquid neck becomes thinner and the lower one breaks up due to the squeezing of airflow $(9.0 \mathrm{~ms})$, circled in red. With the airflow squeezing further, the upper liquid neck breaks up finally $(9.6 \mathrm{~ms})$, which is circled in red. 
Figure 6 shows the velocity field and pressure nephogram $(e=1.0 \mathrm{~mm})$. There are two high-pressure zones at the bottom of liquid film $(9.0 \mathrm{~ms})$, due to which the liquid flows outside and upward around the high-pressure zone. On the other hand, there exists vortexes above the liquid film $(9.0 \mathrm{~ms})$, circled in red, which hinders the liquid film moving upward but promotes flowing sideways, thus a liquid neck forms $(9.6 \mathrm{~ms})$. The airflow and vortexes around the liquid neck become stronger $(9.6 \mathrm{~ms})$, circled in red, causing the liquid neck turns thinner (10.5 ms). Finally, the liquid neck breaks up (10.8 ms).

Figure 7 shows the velocity field and pressure nephogram $(e=2.0 \mathrm{~mm})$. A small high-pressure zone occurs at the bottom of the liquid film $(6.3 \mathrm{~ms})$, circled in red, it can also be observed, the local air flows circumferentially downward along the tube. Due to the axial retraction $(7.2-8.7 \mathrm{~ms})$, a high-pressure zone occurs inside the liquid neck $(8.7 \mathrm{~ms})$. The high-pressure zone hinders the contraction normal to the tube, the corresponding zone becomes thicker $(9.0 \mathrm{~ms})$. Due to the occurrence of the high pressure at the liquid-solid interface, circled in red, the liquid film rebounds away from the tube without break-up (12.3 ms). Combining Figures. 5, 6 and 7, it can be found that the high-pressure zone around the solid-liquid interface moves towards the eccentric side with the increase in eccentric distance.

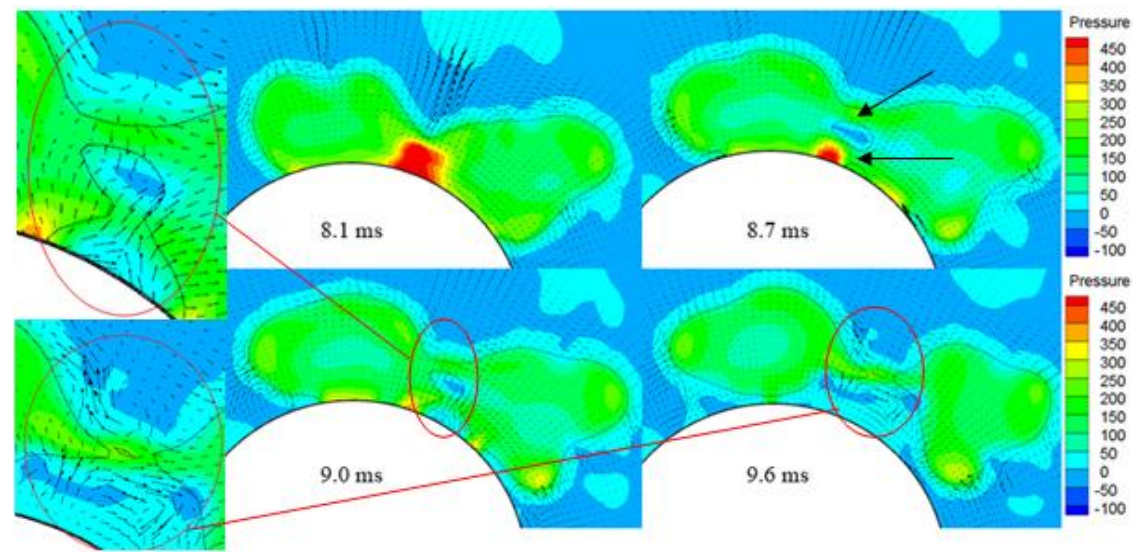

Fig. 5. The velocity field and pressure nephogram during droplet impact $(e=0.5 \mathrm{~mm})$.

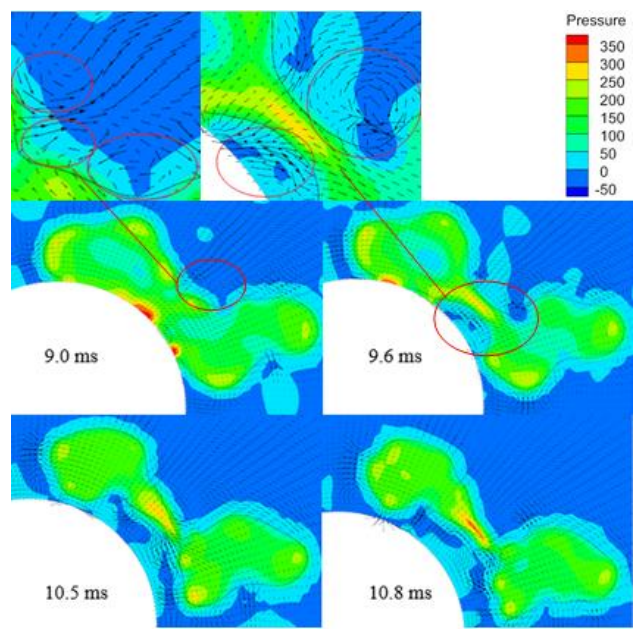

Fig. 6. The velocity field and pressure nephogram during droplet impact $(e=1.0 \mathrm{~mm})$. 


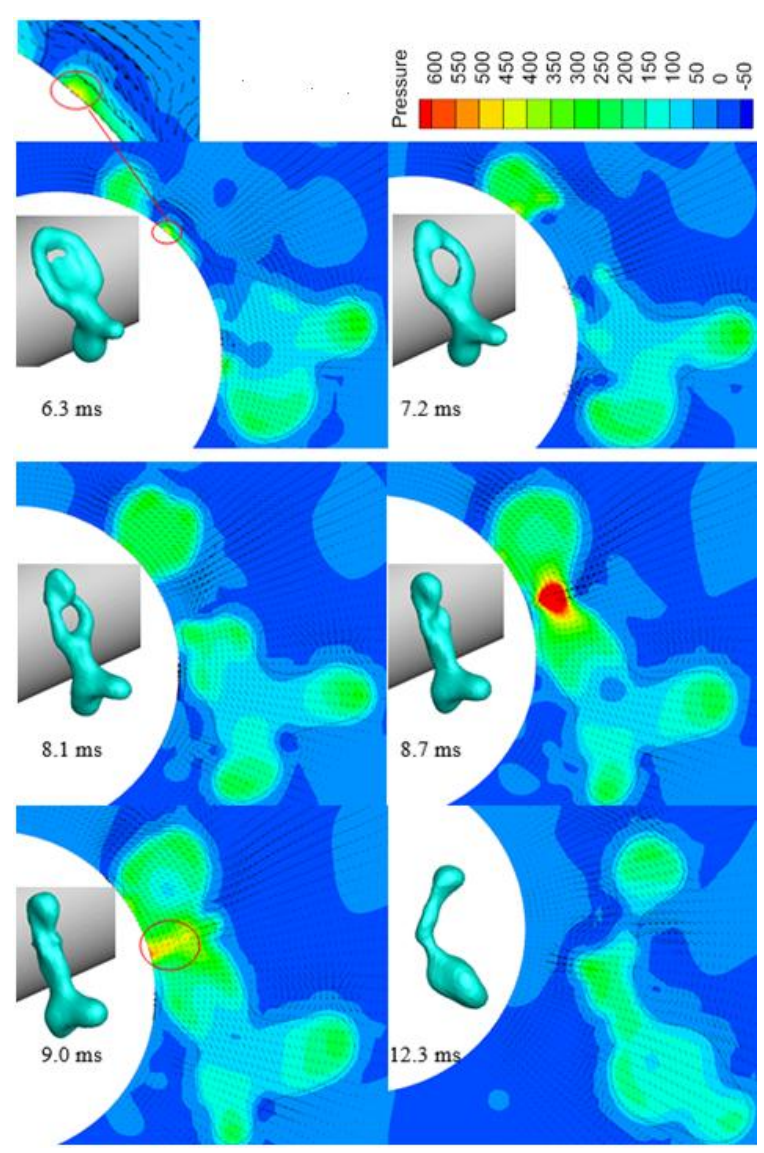

Fig. 7. The velocity field and pressure nephogram during droplet impact $(\mathrm{e}=2.0 \mathrm{~mm})$

\section{Conclusions}

For dual-droplet impacting a super-hydrophobic tube, the influence of eccentric distance on the dynamic characteristics is numerically studied with the CLSVOF method. The eccentric distance hinders the spread during the initial period of spreading. Subsequently, the larger the eccentric distance is, the more the liquid gathers at the eccentric side, which could cause a further spread at the eccentric side later. In addition, the liquid gathering at the rim of liquid film hinders the retraction in the circumferential direction, causing the liquid film retraction in the axial direction is faster than that in the circumferential direction. Besides, the liquid film might be prone to rebound without break-up with the decrease in the eccentric distance, under the same impact velocity. The break-up during rebound mainly depends on local airflow and pressure difference. The high-pressure zone around the solid-liquid interface moves towards the eccentric side with the increase in eccentric distance. 


\section{Acknowledgments}

This work was supported by the Special Project of Liaoning Province for the Central Government to Guide Local Technological Development (No. 2021JH6/10500150) and the National Natural Science Foundation of China (No. 51476017 and 51576029).

\section{References}

[1] M. Rein 1993 Fluid Dyn. Res. 1261

[2] A.L. Yarin 2006 Annu. Rev. Fluid Mech. 38159

[3] M. Marengo, C. Antonini, I.V. Roisman and C. Tropea 2011 Curr. Opin. Colloid Interface Sci. 16292

[4] C. Josserand and S.T. Thoroddsen 2016 Annu. Rev. Fluid Mech. 48365

[5] D. Khojasteh, N.M. Kazerooni and M. Marengo 2019 J. Ind. Eng. Chem. 7150

[6] H. Fujimoto, S. Ito and I. Takezaki 2002 Exp. Fluids. 33500

[7] A.Y. Tong, S. Kasliwal and H. Fujimoto 2007 Num. Heat Trans. 52531

[8] T. Minamikawa, H. Fujimoto and T. Hama 2008 Isij Int. 48611

[9] H. Liu, S.C. Wang, M.Z. Xi, X.J. Jia and Y.L. Wang 2012 J. Jiangsu. 33274

[10]X.Y. Xu, J. Ouyang, T. Jiang and Q. Li 2014 J. Eng. Math. 8535

[11] X.H. Liu, H. Chen, K.M. Wang, S. Chen and S.Q. Shen 2018 J. Therm. Sci. Technol. 17465

[12] K.M. Wang, H. Chen, H.Y. Ge, X.H. Liu, H.S. Liu and S.Q. Shen 2021 Phys. Fluids. 33013301

[13] G.T. Liang, T.Y. Zhang, H.L. Chen, H.B. Yu and S.Q. Shen 2019 Eur. J. Mech. B-Fluids. 74389

[14] Y.L. Guo, L. Wei, S.Q. Shen and G.Y. Chen 2014 Acta Phys. Sin. 63094702

[15] G.T. Liang, T.Y. Zhang, L.Z. Chen, Y. Chen and S.Q. Shen 2019 Int. J. Heat Mass Transfer. 132288

[16] G.T. Liang, T.Y. Zhang, Y. Chen, L.Z. Chen and S.Q. Shen 2019 Int. J. Heat Mass Transfer. 139832

[17]X.H. Liu, K.M. Wang, Y.Q. Fang, R.J. Goldstein and S.Q. Shen 2020 Int. J. Low-Carbon Technol. 15414

[18]X.H. Liu, K.M. Wang, Y.Q. Fang, H. Chen and S.Q. Shen 2020 Exp. Therm. Fluid Sci. 115110060

[19] J.U. Brackbill, D.B. Kothe and C. Zemach 1992 J. Comput. Phys. 100335

[20]H. Chen, X.H. Liu, K.M. Wang, H.S. Liu and S.Q. Shen 2019 Int. J. Comput. Fluid Dyn. 33222

[21] A.S. Grinspana and R. Gnanamoorthy 2010 Colloids Surf., A. 356162

[22]X.H. Liu, Y.M. Zhao, .S. Chen, S.Q. Shen and X.Y. Zhao 2017 Phys. Fluids. 29 062105 